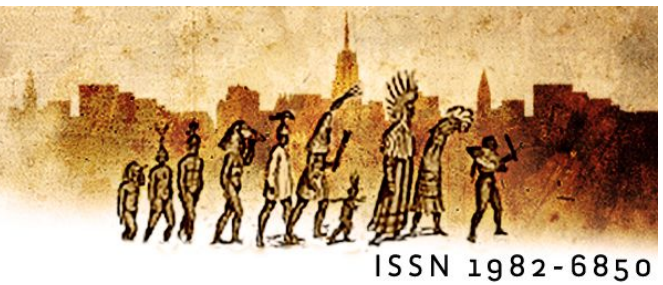

\title{
Postface : \\ Sur quelles bases et comment redynamiser la formation des enseignants ?
}

Jean-Paul Bronckarti (Université de Genève)

Tout d'abord, qu'il me soit permis de remercier les organisatrices de ce numéro, Eulália Leurquin et Fatiha Parahyba, pour avoir sollicité et rassemblé un ensemble de contributions consacrées à la formation des enseignants, dans le cadre de cette troisième présentation, par la revue Eutomia, de travaux inspirés de l'ISD. Ces contributions relatent des recherches abordant des situations et questions de formation initiale ou de formation continue sous des angles d'attaque riches et divers, et fournissent ainsi un très utile tableau des problèmes qui s'y posent, des initiatives qui y sont engagées et des perspectives qui s'y dessinent.

\section{Un positionnement adossé aux principes de I'ISD}

La première caractéristique de ce numéro que je relèverai et saluerai est qu'aucune des contributions qu'il comporte ne se borne à solliciter les seules propositions théoriques ou méthodologiques issues des écrits spécifiques de l'ISD; toutes au contraire, si elles s'inspirent bien évidemment d'éléments de ce courant, font également référence, soit à des auteurs dont les œuvres se situent dans l'amont épistémologique et historique de la démarche de I'ISD, soit à des auteurs et des travaux relevant de courants contemporains compatibles avec ladite démarche.

L'ISD est une approche qui a toujours reconnu sa filiation à l'égard du mouvement rétrospectivement qualifié d'interactionnisme social qui avait pris corps dans le premier tiers du XXe chez des auteurs comme Bühler, Dewey, Mead ou Wallon. L'ISD a toujours 
également proclamé son adhésion plus spécifique aux projets psychologique et éducatif de Vygotski, et s'est profondément inspiré, au travers du cadre épistémologique que s'était donné ce dernier, des écrits philosophiques de Marx et Engels et plus en amont de ceux de Spinoza. L'évocation insistante de ces filiations, à laquelle il convient d'ajouter l'adhésion fondamentale à l'œuvre "réelle" de Saussure (cf. Bronckart, Bulea \& Bota, 2014 ; Bulea, 2013), ne procède pas du geste banal —bien que nécessaire-de "reconnaissance de dettes", mais découle plus profondément de la conviction que les œuvres de ces auteurs constituent des gisements épistémologiques et théoriques largement sous-exploités par les sciences humaines contemporaines, et qu'il est donc nécessaire de les re-convoquer régulièrement pour tenter de bien comprendre les propositions qui y sont formulées et pour donner ce faisant plus de profondeur à nos propres travaux. Dans cette perspective, nous avons fortement apprécié le recadrage marxien des démarches d'analyse du travail proposé dans la contribution d'Alessandra da Silva, Francini Corrêa \& Vera Cristovão, ainsi que la resollicitation des formulations, hypothèses ou principes vygotskiens dont témoignent, outre la contribution ci-dessus citée, celles d'Anderson Carnin, de Glais Cordeiro, de Fatiha Parahyba \& Eulália Leurquin ainsi que de Pollyane Ribeiro \& Fernanda de Castro.

La prise en considération de courants contemporains différents de I'ISD découle de l'acceptation, elle aussi banale ou naturelle, de la nécessité d'une forme de division du travail, c'est-à-dire de répartition des objets ou thématiques à traiter. Elle découle aussi et plus profondément de l'enrichissement des problématiques, des représentations et des interprétations que produisent les interactions attentives avec les divers courants qui sont épistémologiquement compatibles. Parmi ceux évoqués dans ce numéro, on relèvera tout d'abord les courants d'analyse du travail (dont l'analyse du travail enseignant) qu'Anna Rachel Machado et son équipe avaient tôt sollicités (Machado, 2004) et qui avaient également été largement exploités dans les travaux que le groupe LAF (Langage-ActionFormation) avait conduits à Genève (cf. Bronckart, 2008 ; Bronckart \& Groupe LAF, 2004). Comme le montrent plusieurs des contributions à ce numéro, le recours à cette approche, et en particulier aux travaux de Clot, Faïta et Saujat, s'avère productif et enrichissant pour la compréhension du cadre macro-social de l'activité enseignante ainsi que pour la prise en considération de la complexité des strates impliquées dans la mise en œuvre de cette activité et dans la prise de conscience de ses propriétés. Une deuxième zone d'emprunts et d'interactions est constituée par les travaux et propositions émanant de chercheurs 
s'inscrivant dans ce que l'on pourrait aujourd'hui requalifier de didactique générale. II s'agit d'un côté des écrits fondateurs de Chevallard qui ont introduit le concept fameux de transposition didactique, dont les contributions de Glais Cordeiro, de Fatiha Parahyba \& Eulália Leurquin ainsi que de Mariolinda Ferraz \& Adair Gonçalves montrent bien qu'il conserve toute son importance : le phénomène ainsi désigné constitue en effet un élément central de l'activité des enseignants, et dans la mesure où il se déploie en processus complexes et emboîtés, son examen en situation de formation des enseignants est un gage de mise en œuvre d'une profonde réflexion. Il s'agit d'un autre côté de propositions conceptuelles plus récentes, dont la distinction introduite entre les savoirs à enseigner et les savoirs pour enseigner (Hofstetter \& Schneuwly, 2009) qui s'avère utile pour saisir et conceptualiser les difficultés que rencontrent nombre de futurs enseignants en ce qui concerne le statut et l'usage possible de notions présentées au cours de leur formation. On relèvera enfin de nombreuses références à des conceptions de la formation à la lecture où à l'expression textuelle, dont en particulier celle formulée dans les travaux de Kleiman (2008), qui mettent l'accent sur les dimensions d'adéquation sociale et pragmatique des textes plutôt que sur les aspects proprement linguistiques de la généricité ; la sollicitation des écrits de cette auteure (comme des autres écrits de la même tendance) nous parait particulièrement bienvenue dans la mesure où les données empiriques et les réflexions théoriques qu'ils contiennent débouchent sur des logiques de formation auxquelles il est nécessaire et enrichissant de confronter celles qui sont inspirées de l'ISD.

On relèvera encore deux contributions se caractérisant par l'investissement d'un domaine de recherche dans lequel I'ISD était relativement peu actif jusqu'à présent, celui de la lecture. Monica Souza Serafim présente les différents modèles qui y sont en concurrence et relate une étude montrant que les enseignants élaborent une variété de représentations de la lecture correspondant globalement à la variété des positions théoriques. Glais Cordeiro aborde également les représentations de la lecture, mais dans une situation doublement originale, en ce qu'elle porte sur l'éducation précoce et que les représentations sont saisies dans des épisodes d'interaction entre élèves et enseignants. 


\section{La formation des enseignants sous toutes ses coutures}

La plus évidente qualité de ce numéro thématique d'Eutomia est d'aborder la question de la formation des enseignants sous des angles nettement différents, et de fournir ce faisant une image assez complète de la situation en ce domaine. Cependant, l'exhaustivité n'étant, tout comme la perfection, guère du monde des sciences de l'humain, on ne peut prétendre que le tableau ici fourni rende compte de toutes les propriétés de la situation de ces formations. Mais il nous semble néanmoins possible d'y distinguer quatre formes distinctes de saisie de la situation d'un dispositif de formation.

Un premier angle d'attaque consiste en l'examen de l'état des capacités textuelles des enseignants en formation, auquel Fatiha Parahyba \& Eulália Leurquin ont procédé en analysant un ensemble de propriétés linguistiques (en particulier celles relevant des mécanismes de textualisation et des mécanismes énonciatifs) de plusieurs genres académiques produits par les étudiants de deux universités publiques. Cette étude montre que de nombreux étudiants ont de sérieuses difficultés de maitrise discursive, en particulier dans le domaine de la cohérence thématique, ce qui pose évidemment le problème de savoir si, à la fin de leur formation, ils seront aptes à intervenir en ces mêmes domaines auprès de leurs élèves.

Un second angle d'attaque a trait aux représentations se manifestant ou se construisant dans le champ de la formation des enseignants, telles qu'on peut les appréhender dans le cadre d'entretiens dirigés ou semi-dirigés. Alessandra da Silva, Francini Corrêa \& Vera Cristovão ont orienté leurs entretiens sur les représentations que les formateurs d'enseignants ont de leur travail, ce qui a permis de mettre en évidence deux types de difficultés et un point d'ancrage. Les difficultés ont trait d'une part aux résistances et incompréhensions qui se manifestent chez les étudiants, et d'autre part aux limites que ressentent les formateurs quant à leurs propres savoirs et leurs capacités d'adaptation. Le point d'ancrage se situe dans les moyens d'enseignement, vécus par les formateurs comme les principaux instruments sémiotiques dont ils disposent dans leur démarche. Fábio Carneiro \& Lídia Stutz ont de leur côté analysé les représentations construites dans les diários de professeurs de langue en formation initiale ainsi que dans des entretiens tenus avec des professeurs en exercice. Leur analyse de ces documents se centre sur les figures d'action qu'avait conceptualisées Bulea (2010), et confirme un phénomène mis en évidence 
dans les recherches aussi bien brésiliennes (Bulea, Leurquin \& Delano Vidal, 2013) que genevoises (Bulea \& Bronckart, 2010), à savoir le fait que les enseignants passent rapidement de la figuration de leur propre travail à celle de l'activité des élèves, ou encore évaluent leur propre activité à l'aune de ce qu'elle produit sur leurs élèves. Cette étude montre aussi que les savoirs du métier thématisés dans ces productions verbales ont trait aux ressources disponibles, aux procédés d'évaluation et aux stratégies d'enseignementapprentissage. Monica Souza Serafim a comparé les représentations de la lecture construites par un échantillon de professeurs de formation initiale (FI) et un autre de formation continue (FC), recherche qui a fait apparaitre notamment que les professeurs de FI saisissent surtout la lecture dans sa dimension interactive, alors que les professeurs de FC se centrent sur le décodage (avec interprétation unique) des textes. Fatiha Parahyba \& Eulália Leurquin ont quant à elles adressé aux stagiaires un questionnement portant sur les contenus thématiques qui leur paraissent les plus importants, en leur demandant d'en donner les raisons. Les réactions obtenues relèvent plus de la description des expériences vécues que d'une réflexion à caractère professionnel et témoignent en outre d'une confusion entre les savoirs à enseigner et les savoirs pour enseigner.

Un troisième angle d'attaque est constitué de travaux rendant compte de démarches qui pourraient être qualifiées de learning by doing, en ce qu'elles-ci consistent en constructions, par les futurs enseignants, de dispositifs didactiques (en l'occurrence tous centrés sur la dimension de généricité). Mariolinda Ferraz \& Adair Gonçalves rendent compte de la mise en œuvre d'un dispositif de construction, par les formés, de modèles didactiques de genre, dans le cadre d'une démarche collaborative articulant deux types de transposition didactique externe (formation des étudiants et conception de la séquence) et une transposition interne (réalisation de la séquence). Ces transpositions ont été réalisées avec succès et la construction de ces modèles a eu comme bénéfice secondaire que les professeurs on pu prendre conscience de propriétés spécifiques des genres concernés qu'ils n'avaient pas identifiées jusque là. Glais Cordeiro relate quant à elle une démarche d'élaboration d'une séquence d'enseignement portant sur une version du conte du Petit Chaperon Rouge et visant au développement des capacités de compréhension des jeunes enfants ( 4 à 6 ans) concernant en particulier la dynamique des personnages. L'analyse a porté sur les justifications produites par les élèves et a permis de mettre en évidence trois types de verbalisations, allant de la justification très partielle à la justification complète ; 
selon l'auteure, le traitement de ces justifications dans les échanges enseignant/élève constitue un moyen didactique important pour développer les processus de compréhension en lecture des jeunes enfants. Dorotea Kersch \& Renata Marques rendent compte d'un imposant travail ayant trait aux capacités de lecture et d'écriture d'élèves en situation défavorisée et consistant en l'élaboration puis en la mise en œuvre d'un Projet Didactique de Genre sur le thème de la profession Reporter de la communauté, organisé en huit étapes et caractérisé par l'exploitation de différentes technologies digitales. L'analyse du travail produit par un élève a permis de mettre en évidence qu'outre les quatre capacités relevées dans divers travaux de l'ISD (capacités d'action, capacités discursives, capacités linguisticodiscursives et capacités de signification), ce type de projet mobilisait des capacités d'ordre plurisémiotique qui mériteraient de plus amples analyses.

Le quatrième angle d'attaque est celui de l'identification de facteurs et/ou de traces de développement des formés, qu'il s'agisse de développement professionnel ou de développement psychologique personnel. Anderson Carnin a procédé à une étude quasi longitudinale du développement professionnel d'un professeur de langue portugaise qui avait à mettre en œuvre un Projet Didactique de Genre sur le thème Lettre de sollicitation, dans le cadre d'un programme de formation continue. Cette étude a montré que, dans le cadre de l'enseignement ayant trait à la généricité, le concept scientifique de genre pouvait prendre la fonction d'instrument psychologique et contribuer ce faisant à la réorientation de l'agir propre du professeur : le genre constituant ainsi, dans une perspective clairement vygotskienne, un instrument de développement des formateurs autant que des formés. Pollyane Ribeiro \& Fernanda de Castro ont procédé à l'analyse des Rapports de stage initial en tant que genres de texte constituant un lieu révélateur des transformations qui s'opèrent au cours de la formation, ou encore comme lieu dans lequel s'élabore sémiotiquement le monde des activités du sujet. Leur analyse s'est centrée plus particulièrement sur les modalisations en tant que procédés matérialisant et organisant des éléments de représentation d'un futur agir professoral et elle a montré qu'au travers de modalisations déontiques, les rapports de stages d'observation coloraient négativement le regard sur professeur observé, alors le rapport des stages de régence ne présentait pas ce type de modalisation et témoignait d'un processus, nouveau et positif, d'identification avec la profession enseignante. L'étude de Fábio Carneiro \& Lídia Stutz confirme enfin que les figures d'action constituent des traces particulièrement révélatrices de l'état du parcours 
développemental des formés, non seulement par ce qu'elles révèlent de la maitrise discursive de ces derniers, mais surtout par ce qu'elles montrent du cheminement complexe pouvant conduire à une véritable prise de conscience des aspects du métier qui sont de la seule responsabilité de la personne de l'enseignant.

\section{Redynamiser les formations d'enseignants?}

Les travaux réunis dans ce numéro débouchent bien évidemment sur cette question, et I'on peut y lire quelques éléments de réponse que nous reformulerons brièvement.

Certaines contributions soulignent que deux améliorations majeures devraient être apportées aux formations initiales et continues des enseignants, à savoir d'un côté la mise à disposition de temps et d'espaces suffisants pour engager de véritables débats et procéder à une réflexion organisée sur les pratiques didactiques, et d'un autre côté la mise à disposition de moyens d'enseignement qui soient à la fois conformes aux orientations pédagogiques officielles et adaptés aux contexte social réel des établissements scolaires et de leurs élèves.

D'autres contributions insistent sur la nécessité d'un travail visant à la transformation des représentations qu'ont les futurs enseignants des savoirs de leur métier (savoirs à enseigner vs savoirs pour enseigner), ainsi que de la nature même de certains processus d'interaction verbale (concernant en particulier la lecture et la généricité).

D'autres contributions enfin montrent que la voie du learning by doing demeure potentiellement efficace, et que les formations d'enseignants auraient tout à gagner d'une exploitation plus importante des démarches de construction de dispositifs d'enseignement ; et les Projets didactiques de genre constituent à l'évidence un type de dispositif qu'il serait utile de généraliser à d'autres sortes d'objets de l'enseignement du langage.

Ce ne sont certes là que de modestes propositions, mais les éléments de bilan rassemblés dans ce numéro ont eu le mérite de les faire émerger.... et de nous engager toutes et tous à œuvrer à une substantielle amélioration des formations d'enseignants. 


\section{Bibliographie}

BRONCKART, J.-P. O agir nos discursos: das concepções teóricas às concepções dos trabalhadores. Campinas: Mercado de Letras, 2008, 208 p.

BRONCKART J.-P. , BULEA, E. \& BOTA, C. (Ed.) O Projeto de Ferdinand de Saussure. Fortaleza: Parole et Vie, 2014, $434 \mathrm{P}$.

BRONCKART, J.-P. \& Groupe LAF (Ed.) Agir et discours en situation de travail. Cahiers de la Section des sciences de l'Education (Université de Genève), 103, 312 p, 2004.

BULEA, E. Linguagem e efeitos desenvolvimentais da interpretação da atividade. Campinas:

Mercado de Letras, 2010.

BULEA, E. O signo em Ferdinand de Saussure : um campo chave para a chave dos campos. Traduzires, 3, 31-53, 2013. http://seer.bce.unb.br/index.php/traduzires/article/view/9938/7274

BULEA, E. \& BRONCKART, J.-P. Les conditions d'exploitation de l'analyse des pratiques pour la formation des enseignants. Linguarum Arena, 1, 43-60, 2010.

BULEA, E., FRAGA LEUROUIN, E. \& DELANO VIDAL, F. O agir do professor e as figuras de ação: por uma análise interacionista. In L. Bueno, M.-A. Paulino Teixeira Lopes \& V. L. Lopes Cristovão (Ed), Gêneros textuais e formação inicial : uma homenagem à Malu Matencio (pp. 109-132). CampinasSao Paulo : Mercado de letras, 2013.

HOFSTETTER, R. \& Schneuwly, B. (Ed.). Savoirs en transformation: au coeur des professions de l'enseignement et de la formation. Bruxelles: De Boeck, 2009.

KLEIMAN, A. B. A formação do professor. Campinas : Mercado de Letras, 2008.

MACHADO, A.-R. (Org.) O ensino como trabalho: uma abordagem discursiva. Londrina : Eduel, 2004.

\footnotetext{
i Jean-Paul BRONCKART

Faculté de Psychologie et des Sciences de l'Education

Université de Genève
} 